\title{
Editorial: Durability and sustainable concrete
}

\author{
Tom Harrison $\mathrm{BSC}$, PhD \\ Independent consultant and Visiting Industrial Professor, University of
}

Dundee, Dundee, UK

The durability of concrete structures is a major concern for Magazine of Concrete Research, yet in all but rare situations durability is specified by empirical limiting values (e.g. w/c ratio, and minimum cement content). As new constituents arrive on to the market, durability testing is undertaken within a reference concrete (all other constituents being the reference materials) to check if they may be used with the same limiting values. If they pass the test criteria, the constituent is accepted for use within defined limiting values with any other approved constituent.

The concrete sector is under intense pressure to be more sustainable and at the material concrete level this means using less Portland cement clinker, more secondary cementitious materials, recycled water and secondary aggregates. It is a myth that using secondary aggregates always gives a more sustainable solution; for example, if the concrete plant is sitting at its sources of sand and gravel, crushing and transporting recycled concrete aggregates is likely to have a higher environmental impact than using the sand and gravel already at the plant. These laudable pressures on the concrete sector to be more sustainable will not go away.

As a consequence, producers wanting to tick all of the 'green boxes' are producing concretes with cements that have high proportions of secondary cementitious materials and high levels of recycled concrete aggregates. Either of these constituents on their own give concretes with carbonation depths within the normal accepted range but when combined the carbonation depth can be significantly higher than normal. A 'sustainable' concrete must not be at the cost of durability.

Such combinations of constituents should be tested for the relevant aspects of durability and to help with this task, the European committee for the specification of concrete and concrete test methods (CEN/TC 104/SC1 (CEN, 2016) is attempting to produce a suite of durability tests for concrete. These include the following.

- Carbonation in a test chamber at 400 ppm of carbon dioxide $\left(\mathrm{CO}_{2}\right)$, which has been taken as being the approximate natural level of carbon dioxide in rural environments accelerated carbonation test chloride diffusion test
- chloride migration test

- freeze-thaw scaling test

- freeze-thaw internal damage test.

Standardising tests for the chemical resistance of concrete or alkali-aggregate tests has not started at the European level.

This standardisation work is not proving to be an easy task. Experts are very reluctant to move away from their preferred test method because adopting a new method may mean that their data bank of experience may no longer be valid with the different method. There is also an issue over what is the appropriate reference concrete. Traditionally, this has been a Portland cement (CEMI) concrete without admixtures except for freeze-thaw testing. CEN technical report 16563 (CEN, 2013) steers users towards a reference performance in a defined test procedure as this approach removes the uncertainty associated with the reference concrete test value. Where this approach is not taken it recommends that different exposure classes should have different reference concretes and that these concretes are representative of the national/local experience in the defined exposure class.

Nevertheless, progress is being made and one of the tests is published as a full European norm, and two are published as technical specifications, which have the status of drafts for development. None of these tests reflect the reality of the concrete in the structure. They are conventions.

Two of these tests are seen as being input for modelling service life (carbonation in a test chamber and the chloride diffusion test and these tests are regarded as the reference methods) and thus a specified performance in these tests may become requirements for the concrete. This creates a number of difficult and as yet unresolved issues. Is this performance specified as a target value or a characteristic value? If it is a characteristic value, what do you target to ensure a high probability of achieving the characteristic value? Do you simply prove performance by type testing (testing prior to production) and then batch the proven mix proportions using the same sources of constituents? During production do these same mix proportions give the same durability? For how long will the type testing be valid? If the source of the same type and class of constituent is changed, will this have a significant impact on the 
durability? These issues need to be resolved before the producers can start to assess the economics and commercial risks of producing concrete based on a specification of durability by performance.

These two reference tests are expensive and take a long time to complete. Therefore, for practical reasons there is a strong desire to be able to use more accelerated tests, but the relationship between such tests, the reference methods and reality still need to be proven.

There are also unresolved issues with respect to modelling, the main one being for how long does the chloride resistance increase due to ageing? There appears to be no consensus at present.

All this sophistication in testing and specifying concrete must never lose sight of the reality that concrete is made from relatively low cost and variable bulk constituents and that most problems with poor durability are not caused by inadequate specification but by failing to get the basics right; the correct water content and the specified cover to the reinforcement.

The research community have a pivotal role in answering these questions and MCR is keen to publish papers that address these issues.

\section{REFERENCES}

CEN (European Committee of Standardization) (2013)

TR 16563: Principles of the equivalent durability procedure. European Committee of Standardization, Brussels, Belgium.

CEN (2016) CEN/TC104/SC1: Concrete and concrete test methods. European Committee of Standardization, Brussels, Belgium. 